\title{
Theoretical Investigations on Diffusion Induced Thermite Reactions of Core-Shell Aluminum/Palladium Nanoparticles
}

\author{
Ngoc Ha Nguyen ${ }^{1,2}$ and John Z. Wen ${ }^{*}$ \\ ${ }^{1}$ University of Waterloo, Department of Mechanical and Mechatronics Engineering, Waterloo, ON N2L 3G1 Canada \\ ${ }^{2}$ Hanoi National University of Education, Department of Chemistry, Center for Computational Science, Hanoi, Vietnam
}

\begin{abstract}
The bimetallic nanothermites, composed often of the core-shell nanoparticles or the nano-scale multilayers, provide a direct interface between two reactive metals and therefore facilitate the thermite reaction without generating excessive gases. This characteristic is extremely important for wire or material joining applications. In this study we investigated the energetic properties and diffusion characteristics of bimetallic atoms in the Al/Pd core-shell nanoparticles. A classic molecular dynamics approach was implemented using a recently reported force field for the Al/Pd binary system. The results suggested that, in order to preserve the core-shell structure of the $\mathrm{Al} / \mathrm{Pd}$ nanoparticle before being utilized for the material joining purpose, it is essential to maintain an isothermal condition at a lower temperature such as $150 \mathrm{~K}$. In addition, a thicker Pd shell will further hinder the diffusion processes within the core-shell nanoparticle.
\end{abstract}

Keywords: Molecular dynamics, core-shell, nanothermite, diffusion, aluminum, palladium.

\section{INTRODUCTION}

Nanostructured reactive composites have shown their promising applications in localized material and component joining [1]. There are basically two categories of these composites (also called nanothermites), namely, the metastable intermolecular composites (MIC) which contain aluminum nanoparticles and an oxidizer such as $\mathrm{CuO}, \mathrm{Fe}_{2} \mathrm{O}_{3}$, $\mathrm{SnO}_{2}, \mathrm{WO}_{3}$, etc. [2, 3] and the bimetallic energetic nanostructures (e.g., nanoparticles and nanolaminates) of $\mathrm{Ni} / \mathrm{Ti}, \mathrm{Co} / \mathrm{Al}, \mathrm{Ni} / \mathrm{Al}$, and Pt/Al [4]. Recently extensive experimental and numerical studies have been conducted for developing high-efficiency MIC for a variety of purposes. The focus has been on characterizing the reaction kinetics and investigating the interfacial phenomena of the Al nanoparticles and metal-oxide components [2, 3, 5-7]. The important findings from these studies include the revelation of the role of $\mathrm{O}_{2}$ which is generated from decomposition of the oxidizer (e.g., $\mathrm{CuO}$ or $\mathrm{Fe}_{2} \mathrm{O}_{3}$ ) in sustaining the energy propagation rate of thermite reactions [3]. In addition, thanks to the significant energy release from the thermite reactions, the reduced metals are often at their liquid or gaseous states. Table 1 shows the literature date [8] on the adiabatic reaction temperature and the state of products from a set of representative thermite reactions. The formation and existence of gases, whether they are metal vapors or oxygen, hinder the effectiveness of the join process which prefers the direct contact between the adjacent liquid or solid surfaces. The challenge therefore remains in optimizing locally the temperature profile through achieving a careful balance between the energy generation rate from thermite reactions and the heat transfer rate from the reaction site to

*Address correspondence to this author at the University of Waterloo, Department of Mechanical and Mechatronics Engineering, Waterloo, ON N2L 3G1 Canada; Tel: +1 519888 4567; Fax: +1 519885 5862;

E-mail: jzwen@uwaterloo.ca surroundings. In contrary to the MIC, these bimetallic nanothermites, composed often of the core-shell nanoparticles or the nano-scale multilayers, provide a direct interface between two reactive metals and hence facilitate the thermite reaction without generating gases. Table $\mathbf{1}$ shows the thermite reaction between $\mathrm{Al}$ and Pd metals which produces the liquid AlPd alloy under the adiabatic reaction temperature. This characteristic is extremely important for wire or material joining applications in microelectronics and MEMS (microelectromechanical systems) devices where the precisely controlled patterning and manufacturing processes are required.

Table 1. Thermite Reaction Properties of Energetic Materials. The Data were Taken from the Literature [8]

\begin{tabular}{|c|c|c|c|c|}
\hline \multirow{2}{*}{ Reactants } & \multicolumn{2}{|c|}{$\begin{array}{c}\text { Adiabatic } \\
\text { Reaction Temperature (K) }\end{array}$} & \multicolumn{2}{c|}{$\begin{array}{c}\text { State of } \\
\text { Products }\end{array}$} \\
\cline { 2 - 5 } & $\begin{array}{c}\text { w/o } \\
\text { Phase } \\
\text { Changes }\end{array}$ & $\begin{array}{c}\text { with } \\
\text { Phase } \\
\text { Changes }\end{array}$ & $\begin{array}{c}\text { State of } \\
\text { Oxide }\end{array}$ & $\begin{array}{c}\text { State of } \\
\text { Metal }\end{array}$ \\
\hline \hline $\mathrm{Al}+\mathrm{B}_{2} \mathrm{O}_{3}$ & 3995 & 3253 & liquid, gas & gas \\
\hline $\mathrm{Al}+\mathrm{CuO}$ & 5718 & 2843 & liquid & liquid, gas \\
\hline $\mathrm{Al}+\mathrm{Fe}_{2} \mathrm{O}_{3}$ & 4382 & 3135 & liquid & liquid, gas \\
\hline $\mathrm{Al}+\mathrm{NiO}^{2}$ & 3968 & 3187 & liquid & liquid, gas \\
\hline $\mathrm{Al}+\mathrm{SnO}_{2}$ & 5019 & 2876 & liquid & liquid, gas \\
\hline $\mathrm{Al}+\mathrm{WO}_{3}$ & 5544 & 3253 & liquid, gas & liquid \\
\hline $\mathrm{Al}+\mathrm{Pd}$ & 2725 & 2653 & \multicolumn{3}{|c}{ liquid } \\
\hline
\end{tabular}

Few experimental studies have been conducted on the bimetallic nanothermites, although the energies generated through bimetallic thermite reactions are comparable with the energies generated by the MIC (as shown in Fig. 1). A recent work investigated the ignition thresholds and reaction 
velocities of energetic nanolaminates and measured three pairs of metallic components $(\mathrm{Ni} / \mathrm{Ti}, \mathrm{Co} / \mathrm{Al}$, and $\mathrm{Al} / \mathrm{Pt})$ [4]. The literature research on energetic core-shell nanoparticles has mainly focused on synthesis and development of nanostructures $[9,10]$. Detailed investigations on characterizing the reaction properties of these nanostructures have not been widely reported. Meanwhile, theoretical studies, with the help of Molecular Dynamics (MD) simulations, have been actively conducted in predicting the energetic properties of a variety of core-shell nanoparticles [11-17]. While most of these studies focused on Al-Ni binary nanostructures, a recent MD work focused on the thermal stability and energetic reaction properties of palladium coated aluminum nanoparticles at temperatures above $300 \mathrm{~K}$ [18]. That work revealed that for a $5 \mathrm{~nm}$ core-shell nanoparticle, a slower solid-state diffusion of the Al atoms occurs at the low temperature such as $300 \mathrm{~K}$. When the initial temperature was high (such as $600 \mathrm{~K}$ and $700 \mathrm{~K}$ ), a two-stage thermite reaction was observed. At the first stage, the reaction rate was limited by the solid-state diffusion of $\mathrm{Al}$ atoms in the Pd shell. At the second stage when the system temperature was greater than the melting point of the Al core, the reaction rate increased dramatically due to alloying between the liquid $\mathrm{Al}$ core and the $\mathrm{Pd}$ shell. At a much higher temperature such as $900 \mathrm{~K}$, the thermite reaction occurred directly between the liquid $\mathrm{Al}$ core and the Pd shell. The above finding is very important in understanding the reaction mechanism of the $\mathrm{Al} / \mathrm{Pd}$ core-shell nanothermite. It did not reveal the details, however, on the diffusion characteristics of $\mathrm{Al}$ and $\mathrm{Pd}$ atoms which control the structural transformation and energetic performance of nanoparticles at low temperatures. Moreover, the study of diffusion processes is very important for determining the rates of thermite reactions which are induced by the diffusion of corresponding atoms. In addition, the study of diffusion processes within the nanoparticle will help determine the temperature which can preserve the core-shell structures upon their synthesis and before being utilized for the material joining purpose. In this work, we aim at elaborating the diffusion characteristics of bimetallic atoms in the $\mathrm{Al}$ (core)- $\mathrm{Pd}($ shell) nanoparticles at lower temperatures (less than $300 \mathrm{~K}$ ). The controlling parameters such as temperature and reaction time will be evaluated and their influences to the structural transformation and energy release will be quantified using MD simulations. The major characteristics of the solid-state diffusion processes such as the diffusion coefficients of $\mathrm{Al}$ and $\mathrm{Pd}$ atoms will be deduced from these analyses.

\section{MOLECULAR DYNAMICS SIMULATION}

A classic molecular dynamics approach was implemented in this study by means of an open source software GULP [19]. A recently reported force field for the $\mathrm{Al} / \mathrm{Pd}$ binary system was used [18]. This force filed uses the EAM (embedded atom method) potentials which were developed on basis of structural parameters for pure $\mathrm{Al}$ and Pd metals in the literature [20]. In order to investigate the diffusion processes at lower temperatures from $150 \mathrm{~K}$ to $300 \mathrm{~K}$, initially one $\mathrm{Al} / \mathrm{Pd}$ core-shell particle was constructed with a diameter of $5 \mathrm{~nm}$ and consisted of $2243 \mathrm{Al}$ atoms and 2265 Pd atoms. Both metallic atoms were arranged with their fcc phases. The initial nanoparticle structure is shown in Fig. (2). Note that due to the miss-alignment of the core and shell fcc structures it was almost impossible to create a symmetrical structure for the $\mathrm{Al}$ (core) and $\mathrm{Pd}$ (shell) system. Thus as shown, the nanoparticle has a thinner Pd upper layer (zone a with less than two monolayers of Pd atoms) and a thicker Pd bottom layer (zone $\mathbf{b}$ with two or three monolayers of Pd atoms).

In order to study the diffusion processes occurring in the Pd coated Al nanoparticles with four different initial temperatures, MD simulations were conducted for each initial temperature (ranged from $150 \mathrm{~K}$ to $300 \mathrm{~K}$ ) and through the following two-stage procedure. The time step was set to $1 \mathrm{fs}$ for all MD simulation cases.

Step 1: Molecular dynamics relaxation. This step simulates nanoparticle structures at the initial temperatures

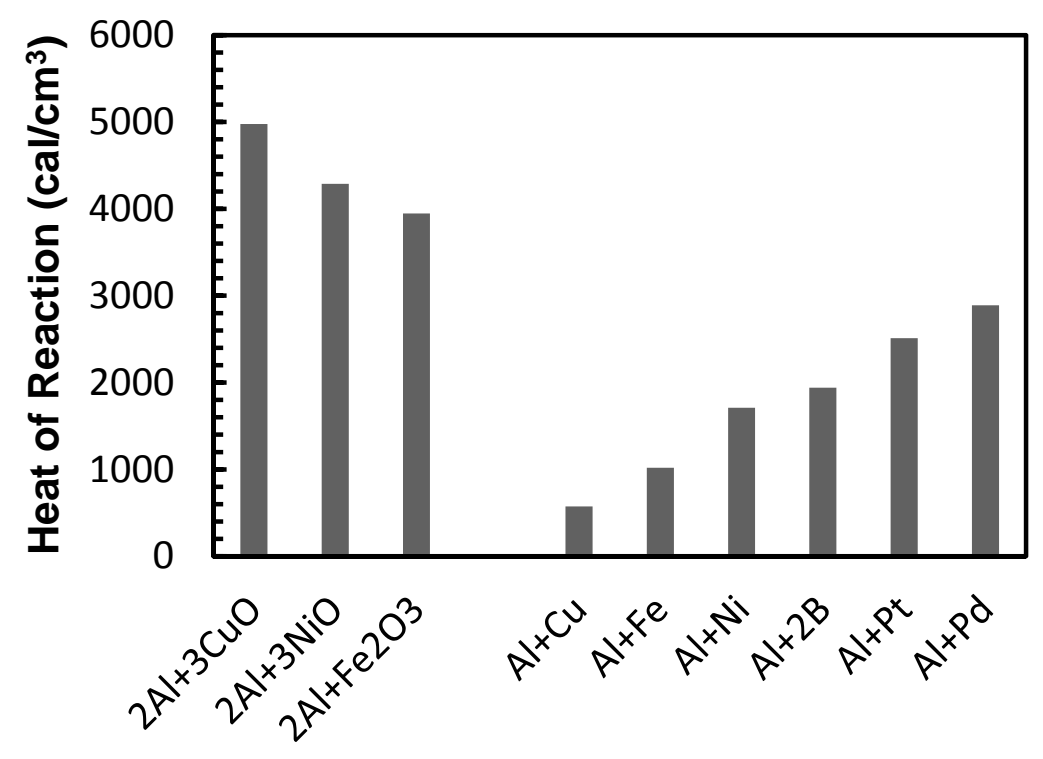

Fig. (1). Heat of reactions from a variety of thermite reactions. 
after a specified buffer period. At this step, the MD simulations were conducted up to $1 \mathrm{ps}$ and the canonical ensemble molecular dynamics (NVT) calculations were used. Totally four initial temperatures (i.e., $150 \mathrm{~K}, 200 \mathrm{~K}$, $250 \mathrm{~K}$ and $300 \mathrm{~K}$ ) were simulated when the temperature kept constant for each run.

Step 2: Molecular dynamics production. This step simulates the diffusion processes and structural changes within the nanoparticles. The simulation time was set to 5000 ps. Both the microcanonical ensemble (NVE) and NVT simulations were conducted for studying diffusion properties of the nanoparticle at varying temperatures. There was no periodic boundary condition applied. Each MD simulation took about 48 hours on a Sharcnet cluster with 256 processors.
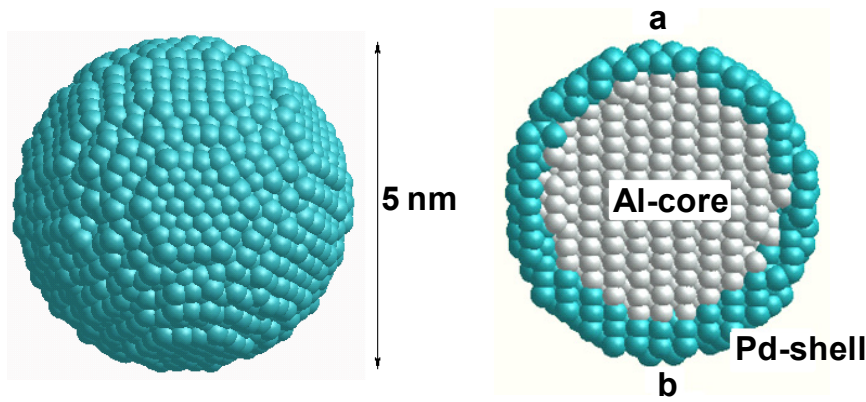

Fig. (2). The initial geometry of the Al/Pd core-shell nanoparticle.

\section{RESULTS AND DISCUSSION}

\section{a) Diffusion Induced Thermite Reactions}

Our previous study suggested that for the Pd coated Al nanoparticles the thermite reactions can be categorized into the diffusion induced exothermic (alloying) reactions during which a clear two-stage mechanism was observed [18]. The alloying process of the bimetallic reactants at their condensed phases (the Al core and the Pd shell in this case) can be described by the following reaction [21].

$$
\mathrm{Al}(\text { core })+\mathrm{Pd}(\text { shell }) \rightarrow \mathrm{AlPd} \text { (alloy) }+\Delta \mathrm{H}
$$

where $\mathrm{Al}$ (core) refers to $\mathrm{Al}$ atoms originated from the core, $\mathrm{Pd}$ (shell) represents $\mathrm{Pd}$ atoms originated from the shell, AlPd (alloy) indicates the newly formed AlPd alloy, and $\Delta \mathrm{H}$ $(<0)$ is the reaction enthalpy (or the enthalpy of formation of AlPd which is the function of temperature). Note that although Reaction 1 is written in the forms of $\mathrm{Al}$ and $\mathrm{Pd}$ atoms, it can be used to describe the propagation rate of the alloying process between the Al metal and Pd metal [21]. In this study, since complete alloying of the core-shell nanoparticles never happens during the MD simulations, Reaction 1 was used to characterize the energetic performance of the diffusion of $\mathrm{Al}$ and $\mathrm{Pd}$ atoms which tends to form the Al-Pd alloy. In order to use Reaction 1, it is essential to identify the phases and energy contents of three participating structures including the reactants (the Al core and the Pd shell) and the product (the AlPd alloy). A recent study [18] suggested that for the AlPd alloy produced from a bimetallic thermite reaction its lattice consists of a space group of Pm3m which satisfies the AlPd B2 phase diagram. In this work, we further elaborate the energy content of this AlPd alloy.

The preliminary studies on basis of classical potential fields failed in predicting the electronic and bonding structures of the target $\mathrm{Al} / \mathrm{Pd}$ nanoparticle. The $a b$ initio approach using the GGA-PBE/Plane-Wave basis set and with ultrasoft pseudopotentials was utilized in this study. The energy cutoff was set to $300 \mathrm{eV}$ and the Brillouin zone of $256 \mathrm{k}$-points in a $8 \times 8 \times 8 \mathrm{k}$-point mesh was chosen. The density of states (DOS), $g(\varepsilon, r)$, is defined as

$$
g(\varepsilon, \mathbf{r})=\sum_{i}\left|\psi_{i}(\mathbf{r})\right|^{2} \delta\left(\varepsilon_{i}-\varepsilon\right)
$$

where $\varepsilon$ is energy, $\mathbf{r}$ is the coordinate, $\varepsilon_{i}$ is the Kohn-Sham eigenvalue corresponding to the Kohn-Sham orbitals $\psi_{\mathrm{i}}(\mathbf{r})$, and $\delta\left(\varepsilon_{i}-\varepsilon\right)$ is delta Dirac function. The partial density of states (PDOS), which is the component of the total density of

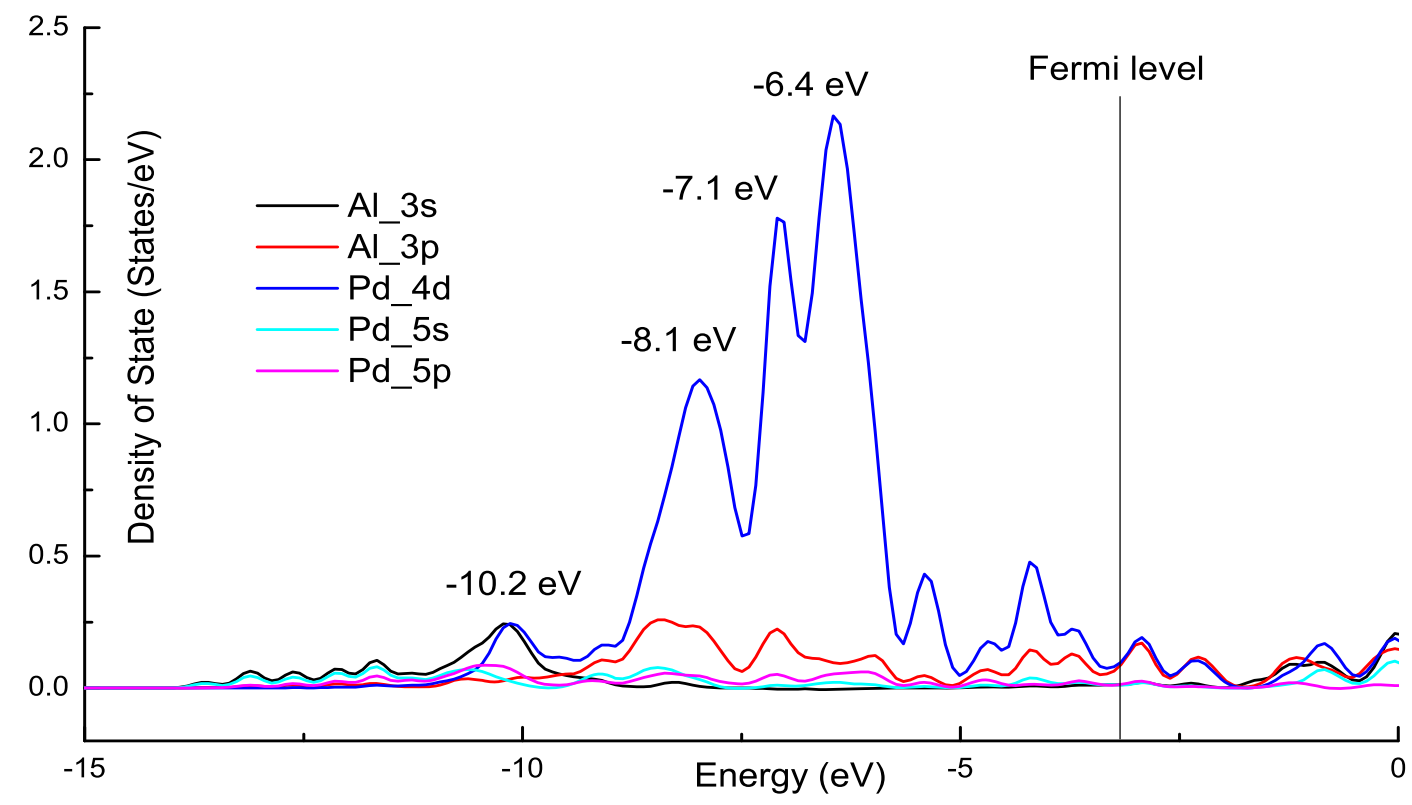

Fig. (3). Total DOS of $\mathrm{Al}$ and $\mathrm{Pd}$ atoms and the AlPd B2 alloy. 
states and shows the energy content of individual orbitals, is defined as

$g_{j}(\varepsilon, \mathbf{r})=\sum_{i}\left|\left\langle\chi_{j} \mid \psi_{i}(\mathbf{r})\right\rangle\right|^{2} \delta\left(\varepsilon_{i}-\varepsilon\right)$

where $\chi_{j}$ is the angular momentum component at each orbital such as $s, p$ or $d$. The DOS calculated for Al and Pd atoms (the reactants) and the AlPd B2 alloy (the product) are shown in Fig. (3).

Clearly in Fig. (3), the DOS peaks of the AlPd B2 alloy at the energy spectra of $-10.2,-8.1,-7.1$ and $-6.4 \mathrm{eV}$ attribute to the occupied states of $\mathrm{Al}$ and $\mathrm{Pd}$ atoms. In addition, these peaks are all below the Fermi level which states the chemical potential of the Al-Pd bond. This indicates that at the AlPd $\mathrm{B} 2$ alloy, the bonds between $\mathrm{Al}$ and $\mathrm{Pd}$ are chemical bonds which are formed from the $\mathrm{Al}$ core and the $\mathrm{Pd}$ shell and through Reaction 1.

Fig. (4) shows the PDOS calculated for each state of Al and $\mathrm{Pd}$ atoms in the AlPd B2 alloy. It further reveals, the DOS peak of the AlPd alloy at $-10.2 \mathrm{eV}$ results from the overlap between the $4 \mathrm{~d} \mathrm{Pd}$ and $3 \mathrm{~s}$ Al orbitals while the DOS peaks at -8.1 and $-7.2 \mathrm{eV}$ attribute to the overlap between the $4 \mathrm{~d} \mathrm{Pd}$ and $3 \mathrm{p} \mathrm{Al}$ orbitals. The above $a b$ initio calculation derives an energy release $(\Delta \mathrm{H})$ of $-175.7 \mathrm{~kJ} /(\mathrm{mol} \mathrm{AlPd})$ from Reaction 1. This value agrees well with the previously reported data $(-182.5 \pm 9.4 \mathrm{~kJ} / \mathrm{mol})$ [22]. This energy release, which is calculated for bulk metals and with a periodic boundary condition applied, is different from the energy release generated from the thermite reaction of the core-shell nanoparticle.

\section{b) Energetic Properties of the Diffusion Processes}

If the energetic behavior of Reaction 1 is characterized under the adiabatic condition, the total energy (i.e., the summation of the kinetic and potential energies of the Al core, Pd shell, newly formed AlPd alloy and the heat of reaction) should be conserved. During the process, the energy release from Reaction $1(\Delta \mathrm{H})$ will cause the temperature increase and subsequently the change in the thermal kinetic energy of the nanoparticle. Table $\mathbf{2}$ shows the changes in the system kinetic energy $\Delta \boldsymbol{K} \boldsymbol{E}$ and the temperature increasing rates from the NVE (adiabatic) simulations. Four nanoparticles with different initial temperatures were simulated over the specified period (5000ps). Fig. (5) shows the MD calculated temperature profiles for four processes indicated in Table 2.

Table 2. The Changes in Kinetic Energy $\Delta K E$ (in $\mathbf{J} / \mathbf{m o l}$ AIPd) and Temperature Increasing Rates $d T / d t$ (K/Picosecond) for Four Nanoparticles Initiated at Different Temperatures (in K)

\begin{tabular}{|c|c|c|c|c|}
\hline Initial Temperature & $\mathbf{1 5 0}$ & $\mathbf{2 0 0}$ & $\mathbf{2 5 0}$ & $\mathbf{3 0 0}$ \\
\hline \hline$\Delta K E$ & 1728.5 & 2679.8 & 4040.0 & 5193.5 \\
\hline$d T / d t$ & 0.0127 & 0.02 & 0.0314 & 0.0314 \\
\hline
\end{tabular}

Fig. (5) shows that, when the initial temperature is low, such as at $150 \mathrm{~K}, 200 \mathrm{~K}, 250 \mathrm{~K}$ and $300 \mathrm{~K}$, the system temperature of the $\mathrm{Al} / \mathrm{Pd}$ core-shell nanoparticle increases with the reaction time. And, more importantly, the temperature first increases with a linear function of the reaction time within the simulation period (up to $3500 \mathrm{ps}$ ). After $3500 \mathrm{ps}$, a stage with the accelerated temperature increase is observed for the higher temperature (such as $250 \mathrm{~K}$ and $300 \mathrm{~K}$ ). Both Fig. (5) and Table 2 show, the higher the initial temperature is, the greater change in kinetic energies (shown as the different temperature increase for different cases) will be derived. And the greatest temperature slope was observed for the initial temperature of $300 \mathrm{~K}$. This suggests the larger diffusion rates of $\mathrm{Al}$ and $\mathrm{Pd}$ atoms at

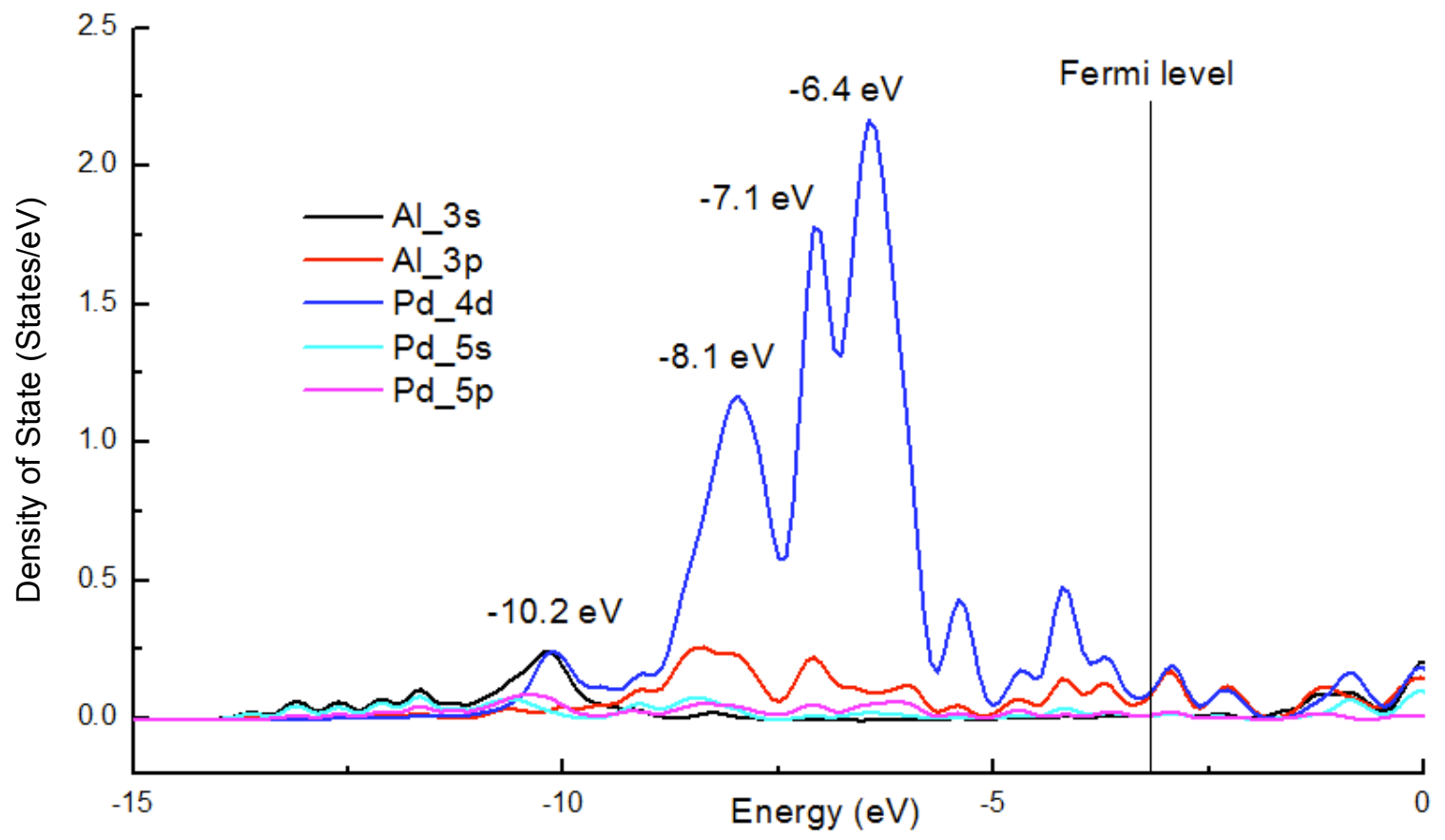

Fig. (4). PDOS of each state of $\mathrm{Al}$ and $\mathrm{Pd}$ atoms in the AlPd B2 alloy. 


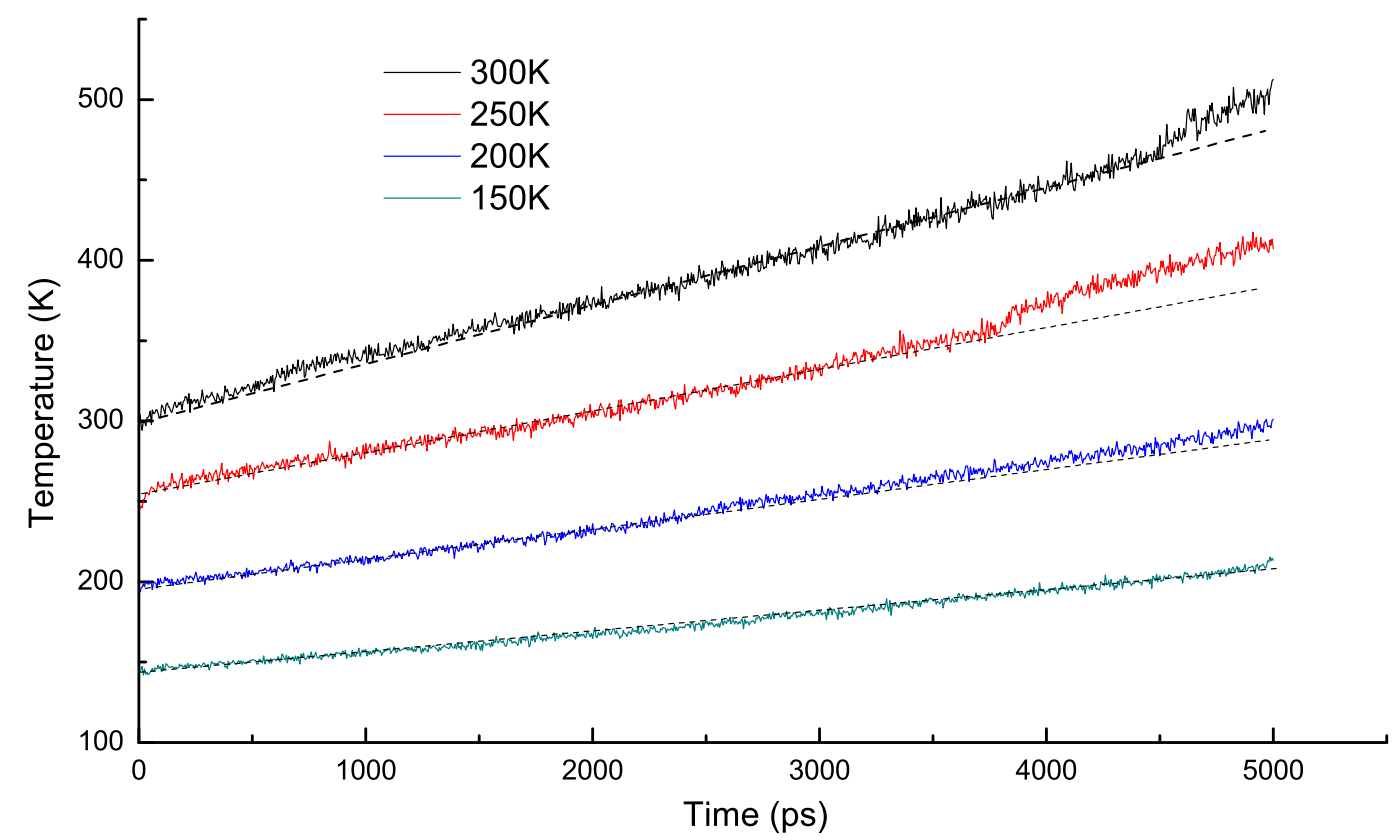

Fig. (5). The MD calculated temperature profiles of the Al/Pd core-shell nanoparticle with different initial temperatures. The dash lines are plotted to show the linear functions.

higher initial temperatures. It is easy to expect that, with the increasing temperature of the nanoparticle, the melting point of the Al core will be first reached and the thermite reaction between $\mathrm{Al}$ and $\mathrm{Pd}$ will follow. This confirms a shorter ignition period of the core-shell nanoparticles when the initial temperature is higher.

\section{c) Characteristics of Diffusion Processes within the Al/Pd Core-Shell Nanoparticle}

In order to characterize the diffusion process within the core-shell nanoparticles, the pair correlation function $\mathrm{g}(\mathrm{r})$, which is defined as the probability of finding the center of a particle (e.g., Pd atom) for a given distance from the center of the other particle (e.g., Al atom), was calculated using the method shown in literature [23]. Fig. (6) shows the $g(r)$ functions calculated at $5000 \mathrm{ps}$ for different initial temperatures. During these simulations, the adiabatic condition (NVE) was applied. Fig. (6) shows for all four initial temperatures three major peaks at 2.69, 4.81 and 7.39 $\AA$ exist and refer to the heterogeneous structures of the $\mathrm{Al}$ core and the $\mathrm{Pd}$ shell. Note that the lattice constants for fcc aluminum and palladium are $4.05 \AA$ and $3.89 \AA$,

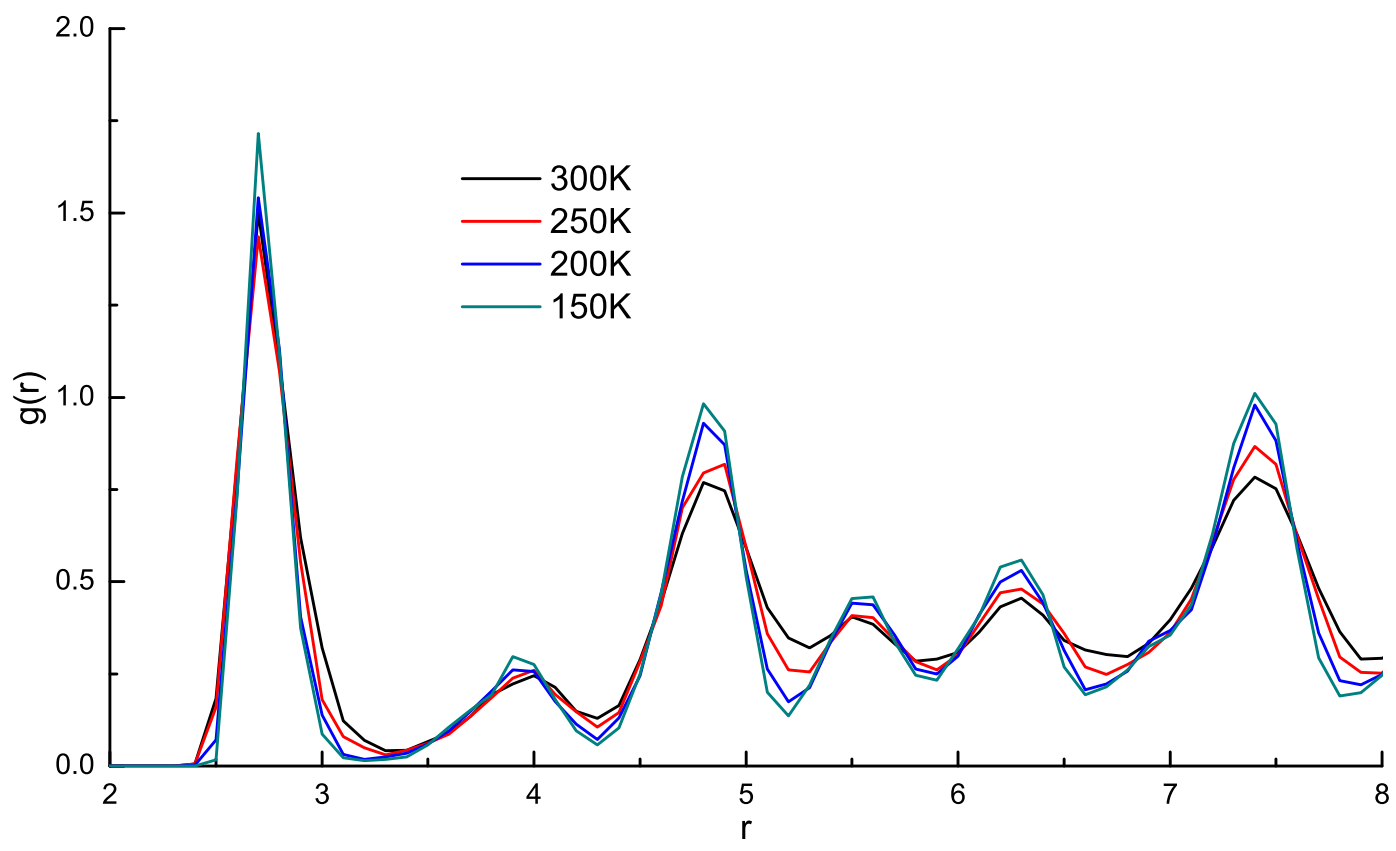

Fig. (6). The pair correlation function calculated for the A1/Pd core-shell nanoparticle in NVE at differently initial temperatures after 5000 ps. The distance $r$ is in $\AA$. 
(a)

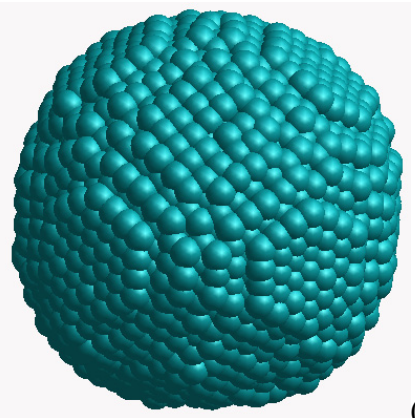

(b)

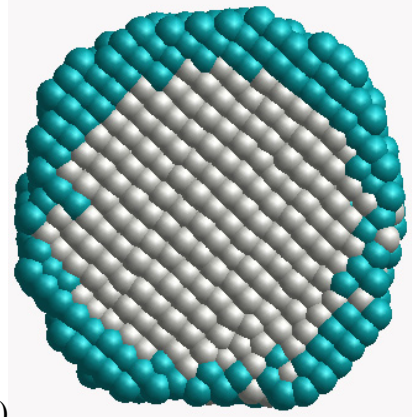

(c)

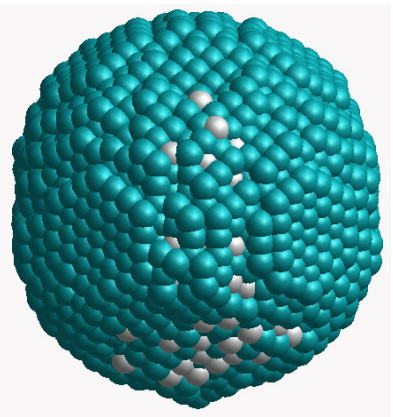

Fig. (7). Snapshots of the Al core and Pd shell predicted during the NVE simulation for the initial temperature of $300 \mathrm{~K}$ at 0 ps (a) and after 5000 ps: (b) cross-section view; (c) surface view.

respectively. The sharper distributions of $g(r)$ at lower temperatures confirmed the more distinguishable core-shell structures at these temperatures. Fig. (7) shows the snapshots of the core-shell structure predicted at 5000 ps for the initial temperature of $300 \mathrm{~K}$. Clearly, both the core and the shell remain their crystalline structures though a few Al atoms diffused onto the surface of the Pd shell while some Pd atoms diffused in the $\mathrm{Al}$ core. In comparison with the temperature profile shown in Fig. (4), these diffusion processes of $\mathrm{Al}$ and $\mathrm{Pd}$ atoms result in a temperature increase of $175 \mathrm{~K}$ over $5000 \mathrm{ps}$.

When the MD simulations were carried out under the isothermal condition (NVT), the $\mathrm{g}(\mathrm{r})$ curves shows more comparable distributions for all four temperatures, as observed in Fig. (8). The comparison between Figs. $(\mathbf{6}, \mathbf{8})$ shows that, under the isothermal condition, the three major peaks exhibit higher probabilities and the differences in the $\mathrm{g}(\mathrm{r})$ among four temperatures become more uniform. This implies, in order to keep the core-shell structure, an isothermal condition should be established.

The diffusion pathways of $\mathrm{Al}$ and $\mathrm{Pd}$ atoms within the core-shell nanoparticle can be further characterized using their mean square displacements (MSD) which are calculated by tracking individual atoms. The MSD are defined as

$$
M S D=\left\langle r^{2}(t)\right\rangle=\left\langle\frac{1}{N} \sum_{i=0}^{N}\left(r_{i}(t)-r_{i}(0)\right)^{2}\right\rangle
$$

where $N$ is the number of atoms (Al or $\mathrm{Pd}), t$ corresponds to time, and $r_{i}(t)-r_{i}(0)$ is the vector distance traveled by a given particle over the time interval $t$. The MSD were calculated for the $\mathrm{Al}$ and $\mathrm{Pd}$ atoms, respectively, for the specified temperature. The MSD curves provide information on the diffusivities of $\mathrm{Al}$ and $\mathrm{Pd}$ atoms. General speaking, if the system is liquid, the MSD curve grows linearly with the reaction time. When the system is solid, however, the MSD curve trends to and saturates at a finite value. After the MSD curve is plot as the function of the reaction time, the slope of this curve in a linear region can be used to characterize the diffusion coefficient of the corresponding atom. The correlation between the MSD and the diffusion coefficient D can be written as

$$
\frac{\partial(M S D)}{\partial t}=2 d D
$$

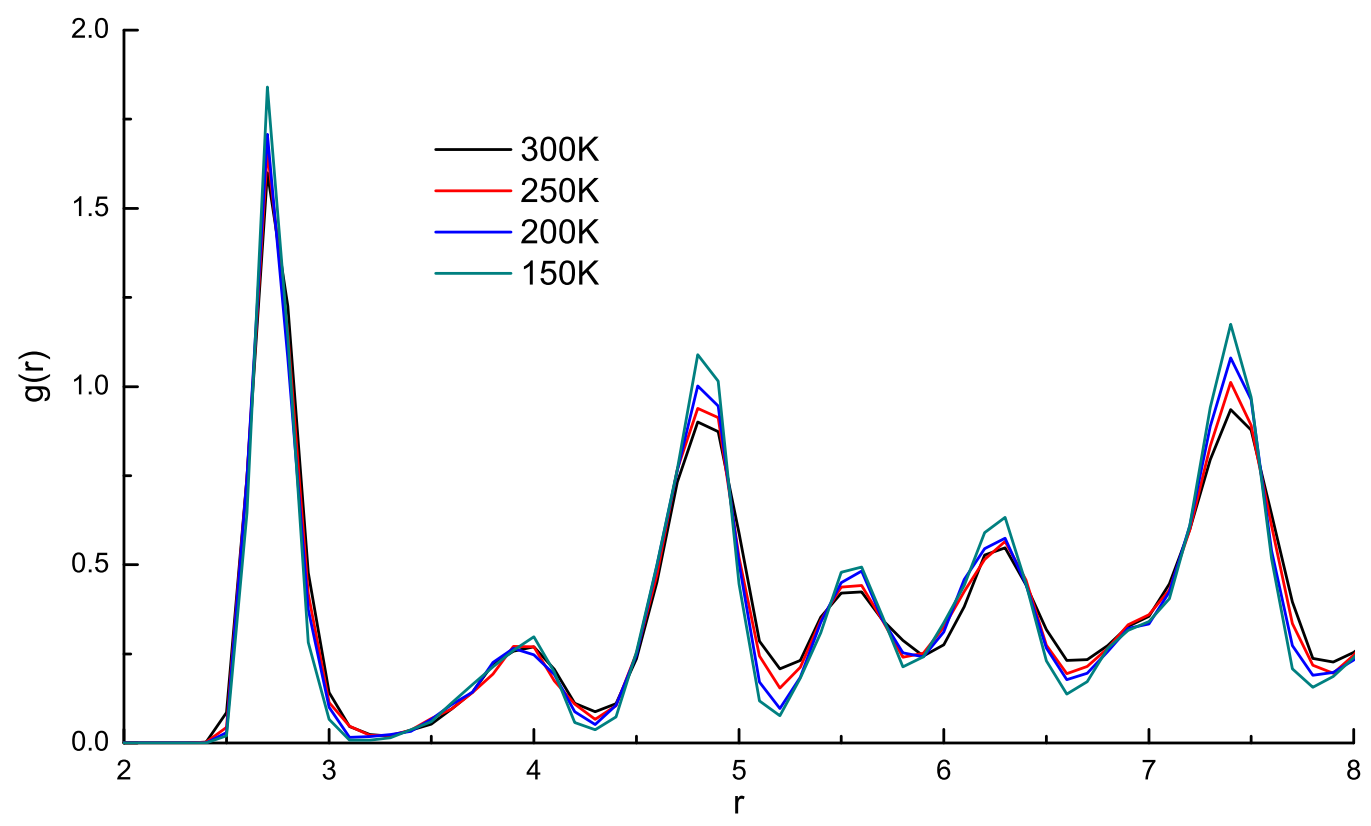

Fig. (8). The pair correlation function calculated for the A1/Pd core-shell nanoparticle in NVT at differently initial temperatures after 5000 ps. 
where $d$, the number of system dimensions, is 3 for an overall diffusion, and 1 for a radial diffusion [24]. In this study, the radial diffusion has been applied to describe the diffusion of $\mathrm{Al}$ atoms in the $\mathrm{Pd}$ shell and the reverse process of $\mathrm{Pd}$ atoms in the $\mathrm{Al}$ core. The diffusion coefficient $D$ [length ${ }^{2} /$ time] in Equation 5 can be written as

$D=D_{0} e^{-Q / R T}$

where $D_{0}$ is the maximum diffusion coefficient [length ${ }^{2} /$ time], $Q$ is the activation energy of the diffusion [energy/mol], $T$ is the absolute temperature [K], and $R$ is the gas constant [energy/(temperature*mol)].

Under the adiabatic condition with the NVE simulations, the MSD calculated for representative temperatures are shown in Fig. (9). Because during the simulation period the particle temperature increases with time, the diffusion coefficients of $\mathrm{Al}$ and $\mathrm{Pd}$ atoms are both changing. It is interesting to observe that, the MSD of Pd atoms are always larger than the values of $\mathrm{Al}$ atoms. This can be explained by the higher energy content of $\mathrm{Pd}$ atoms than $\mathrm{Al}$ atoms at the same temperature. This will be investigated in detail using the following NVT (isothermal) simulations.

Fig. (10) shows the MSD calculated at isothermal conditions (NVT) for both $\mathrm{Al}$ and $\mathrm{Pd}$ atoms within four different nanoparticles. Generally speaking, for a given temperature, the MSD of $\mathrm{Pd}$ atoms is always higher than that of $\mathrm{Al}$ atoms. This can be explained by a larger energy content (mainly the potential energy) of $\mathrm{Pd}$ atoms than that for $\mathrm{Al}$ atoms for the simulation period. In addition, it can be observed from Fig. (10) that, sharp jumps of the MSD from 0 ps occur at the beginning of all four simulations. This behavior results from the self-adjustment of the core-shell nanoparticle following the step 1 buffer period. Note that because the core-shell structure preserves over the entire MD simulation period, the radial gradients of both $\mathrm{Al}$ and $\mathrm{Pd}$ atom concentrations remain almost same (although the maximum gradient exists at the core-shell interface). This implies, according to the Fick's law, the change in MSD values is predominately determined by the diffusion coefficients of these $\mathrm{Al}$ and $\mathrm{Pd}$ atoms. Following the selfadjustment of the system, for a lower temperature such as $150 \mathrm{~K}$, both $\mathrm{Al}$ and $\mathrm{Pd}$ atoms exhibit the flat MSD curves which show their negligible diffusion coefficients at this temperature. When the temperature is $200 \mathrm{~K}$, the selfadjustment periods for both $\mathrm{Al}$ and $\mathrm{Pd}$ atoms last longer and are followed by the flat curves. When the temperatures are $250 \mathrm{~K}$ and $300 \mathrm{~K}$, the two-stage MSD curves can be observed for both $\mathrm{Al}$ and $\mathrm{Pd}$ atoms. A similar process was observed in a previous study when a sintering process between two nanoparticles was investigated [25]. For this temperatureindependent MSD behavior, the first stage (shown as the flat line for less than 2000 ps for $300 \mathrm{~K}$ ) corresponds to the lowamplitude vibration of $\mathrm{Al}$ and $\mathrm{Pd}$ atoms about their own lattices. Then a relaxation process occurs due to the diffusion of $\mathrm{Al}$ atoms into the $\mathrm{Pd}$ shell and the diffusion of $\mathrm{Pd}$ atoms into the Al core (shown as the linear line with a slope). These processes end quickly when the localized gradients of atom concentrations are not big enough to drive a significant mass flux. At the second stage (shown as the flat line for more than $2500 \mathrm{ps}$ ) the diffusion of $\mathrm{Al}$ and $\mathrm{Pd}$ atoms become insignificant and atoms vibrate about their own lattices again. The observation further confirms, when the temperature increases, the degree of atom vibrations become greater (shown as the enlarged change in MSD amplitudes about the top flat line). By using Equation 5, the atom diffusion processes in the core-shell nanoparticle can be $\AA^{2} / \mathrm{ps}$ and $7.335 \times 10^{-5} \AA^{2} / \mathrm{ps}$ for $250 \mathrm{~K}$ and $300 \mathrm{~K}$, respectively. For $\mathrm{Pd}$ atoms, the diffusion coefficients are $8.856 \times 10^{-5} \AA^{2} / \mathrm{ps}$ and $2.063 \times 10^{-4} \AA^{2} / \mathrm{ps}$ for $250 \mathrm{~K}$ and $300 \mathrm{~K}$, respectively. It is worthwhile to mention that, these diffusion coefficients refer to the mean coefficients of all participated atoms in the nanoparticle (Al or $\mathrm{Pd})$. The $\mathrm{Al}$

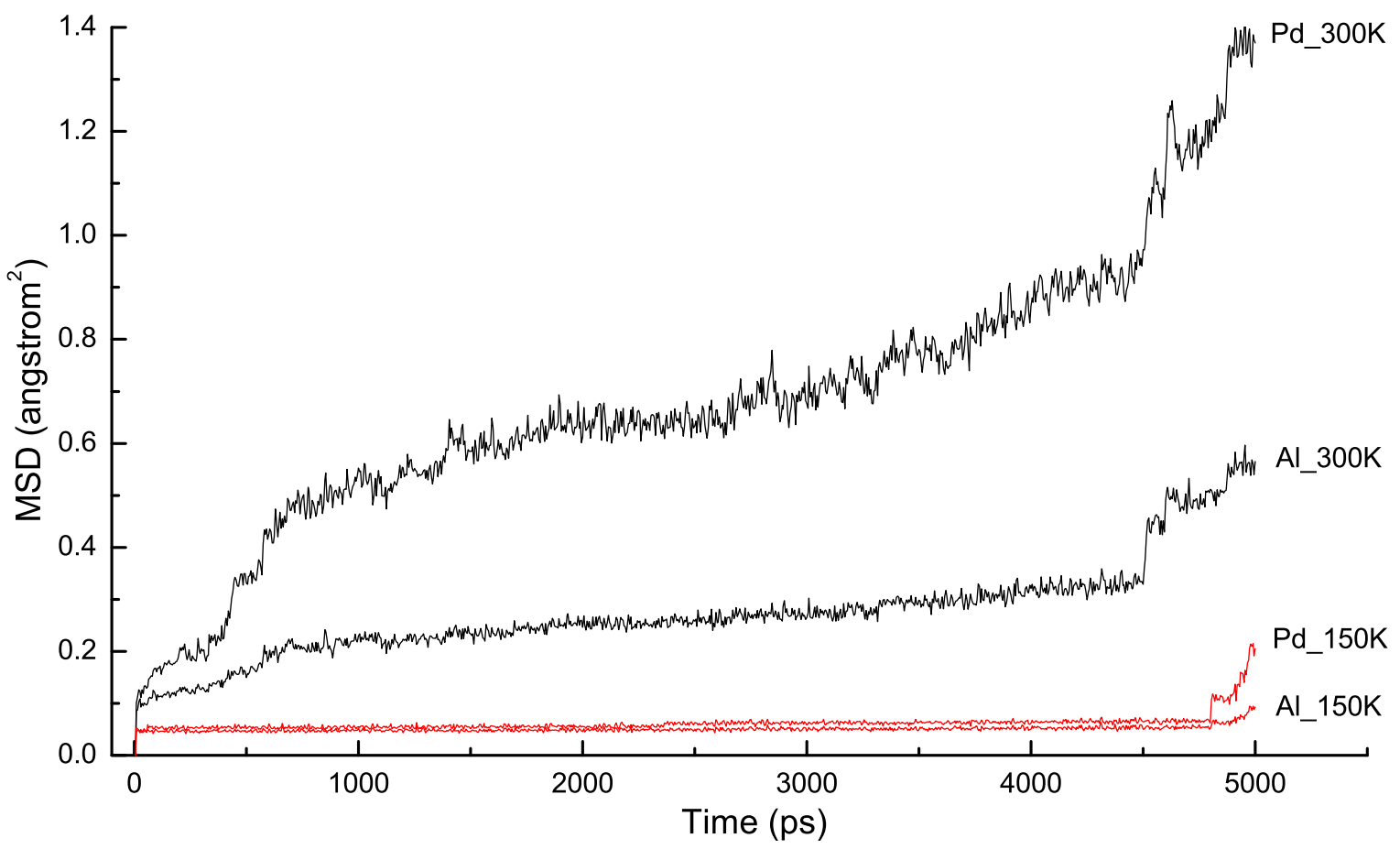

Fig. (9). $M S D$ of the $\mathrm{Al} / \mathrm{Pd}$ core-shell nanoparticle in NVE simulations. 


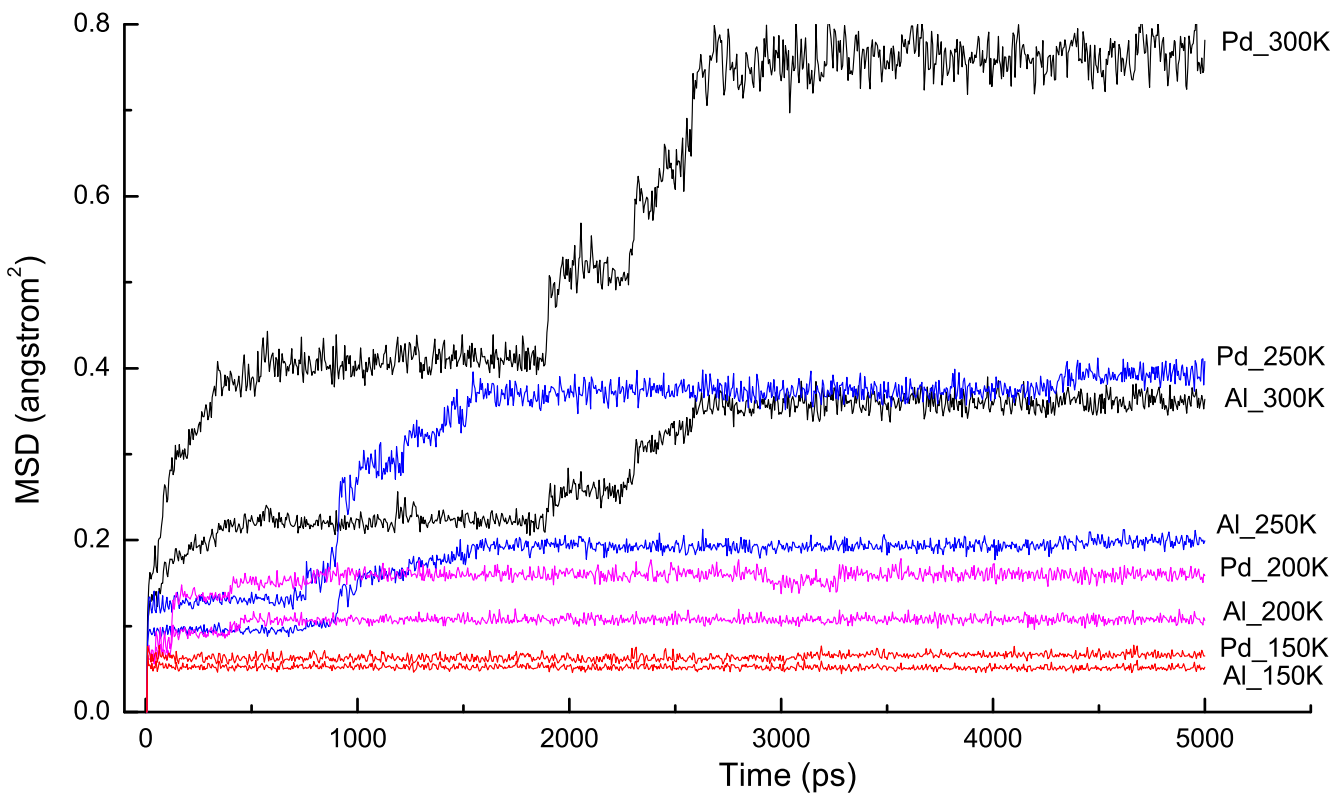

Fig. (10). $M S D$ of the $\mathrm{Al} / \mathrm{Pd}$ core-shell nanoparticle in NVT simulations.

atoms at the Al-Pd interface may have a much larger diffusivity comparing to the $\mathrm{Al}$ atoms in the core.

It is interesting as well to compare the energy contents of the $\mathrm{Pd}$ shell and the Al core. As shown in Fig. (10), the Pd atoms in the shell have the greater mobility to move away from their lattices and towards the core and the outer space. The initial structure of the nanoparticle shows a Pd shell with the mean thickness of $4.3 \AA$ and the radius of the Al core is $20.7 \AA$. Based on these values, the surface areas $(S)$ of the Al core and the Pd shell can be calculated as $5384.6 \AA^{2}$ and $13238.6 \AA^{2}$, respectively. Given the bulk energy of the nanoparticle as $E_{B u l k}$ and the total energy for the nanoparticle with the surface as $E_{\text {Surface }}$, the surface energy $E_{S}$ is then defined as

$E_{S}=\frac{E_{\text {Surface }}-E_{\text {Bulk }}}{S}$

The MD simulations provided the energy data for Equation 7 and Table 3 shows the results.

Table 3. The Calculated Energy Data: $\boldsymbol{E}_{\text {Bulk }}$ and $\boldsymbol{E}_{\text {Surface }}$ are in $\mathrm{eV}, S$ is in $\AA^{2}$ and $E_{S}$ is in $\mathrm{eV} / \AA^{2}$

\begin{tabular}{|c|c|c|c|c|}
\hline & $\boldsymbol{E}_{\text {Bulk }}$ & $\boldsymbol{E}_{\text {Surface }}$ & $\boldsymbol{S}$ & $\boldsymbol{E}_{\boldsymbol{S}} \cdot \mathbf{1 0}^{\mathbf{2}}$ \\
\hline \hline $\mathrm{Al}(2243$ atoms) & -7498.409 & -7180.461 & 5384.6 & 5.9 \\
\hline $\mathrm{Pd}(2265$ atoms) & -8915.910 & -7990.263 & 13238.6 & 7.0 \\
\hline
\end{tabular}

Table 3 shows the larger surface energy per unit area of the $\mathrm{Pd}$ atoms than that of the $\mathrm{Al}$ atoms. The data in Table $\mathbf{3}$ can be further used to calculate the total energy contents (as the product of $S$ and $\left.E_{S}\right)$ of the Pd surface $(926 \mathrm{eV})$ and the Al-Pd interface $(318 \mathrm{eV})$. The much greater surface energy of the Pd shell shows its larger instability and subsequently the greater mobility. Because the surface energy becomes smaller when the nanoparticle size becomes larger and for the Pd shell the larger thickness will significantly reduce the instability of Pd atoms, a thicker Pd shell is preferred for the $\mathrm{Al} / \mathrm{Pd}$ core-shell nanoparticles in order to preserve their coreshell structures.

\section{CONCLUSIONS}

A classic molecular dynamics approach was implemented in this study in order to investigate the diffusion processes of $\mathrm{Al}$ and $\mathrm{Pd}$ atoms within one $5 \mathrm{~nm}$ $\mathrm{Al} / \mathrm{Pd}$ core-shell nanoparticle. The simulations were conducted in adiabatic (NVT) and isothermal (NVE) conditions and up to $5000 \mathrm{ps}$. The energetic performance of the bimetallic thermite reaction was studied and the diffusion coefficients of $\mathrm{Al}$ and $\mathrm{Pd}$ atoms within the core-shell nanoparticle were derived. The MD simulations revealed a higher energy content of the Pd shell in comparison with the Al core at the same temperature. When the adiabatic condition was applied, the system temperature kept increasing even the initial temperature was set at $150 \mathrm{~K}$. The results further suggested that, in order to preserve the coreshell structure of the Al/Pd nanoparticle before being utilized for the material joining purpose, it is essential to maintain an isothermal condition at a lower temperature such as $150 \mathrm{~K}$. In addition, a thicker Pd shell will further hinder the diffusion processes within the core-shell nanoparticle.

\section{ACKNOWLEDGEMENTS}

We would like to thank Dr. Le Minh Cam and Dr. John Persic for helpful discussions. This project was supported by Natural Sciences and Engineering Research Council of Canada.

\section{REFERENCES}

[1] Rossi C, Zhang K, Esteve D, Alphonse P, Tailhades P, Vahlas C. Nanoenergetic materials for MEMS: A review. J Microelectromech Syst 2007; 16: 919-31.

[2] Sullivan K, Zachariah MR. Simultaneous pressure and optical measurements of nanoaluminum thermites: investigating the reaction mechanism. J Propul Power 2010; 26: 467-72.

[3] Zhou L, Piekiel N, Chowdhury S, Zachariah MR. Time-resolved mass spectrometry of the exothermic reaction between 
nanoaluminum and metal oxides: the role of oxygen release. J Phys Chem C 2010; 114: 14269-75.

[4] Picard YN, McDonald JP, Friedmann TA, Yalisove SM, Adams DP. Nanosecond laser induced ignition thresholds and reaction velocities of energetic bimetallic nanolaminates. Appl Phys Lett 2008; 93: 104104 .

[5] Apperson S, Shende RV, Subramanian S, et al. Generation of fast propagating combustion and shock waves with copper oxide/aluminum nanothermite composites. Appl Phys Lett 2007; 91: 243109 .

[6] Cheng JL, Hng HH, Ng HY, Soon PC, Lee YW. Synthesis and characterization of self-assembled nanoenergetic Al-Fe2O3 thermite system. J Phys Chem Solids 2010; 71: 90-4.

[7] Zhang K, Rossi C, Rodriguez GAA, Tenailleau C, Alphonse P. Development of a nano- $\mathrm{Al} / \mathrm{CuO}$ based energetic material on silicon substrate. Appl Phys Lett 2007; 91: 113117.

[8] Fischer SH, Grubelich MC. Theoretical energy release of thermites, intermetallics, and combustible metals. Sandia National Laboratories. Albuquerque NM 1998.

[9] Cheng ZP, Li FS, Yang Y, Yang YL, Liu XD. A new approach to synthesize core-shell nanosized Ni/Al composite powder. Rare Met Mater Eng 2007; 36: 713-6.

[10] Cheng ZP, Yang Y, Liu XD, Yang YL, Li FS. Preparation of coreshell $\mathrm{Cu} / \mathrm{Al}$ powders by displacement method. Acta Chim Sinica 2007; 65: 81-5.

[11] Delogu F. Numerical simulation of the thermal response of $\mathrm{Al}$ core/Ni shell nanometer-sized particles. Nanotechnology 2007; 18: 505702.

[12] Evteev AV, Levchenko EV, Riley DP, Belova IV, Murch GE. Reaction of a Ni-coated Al nanoparticle to form B2-NiAl: A molecular dynamics study. Philos Mag Lett 2009; 89: 815-30.

[13] Levchenko EV, Evteev AV, Riley DP, Belova IV, Murch GE. Molecular dynamics simulation of the alloying reaction in Alcoated Ni nanoparticle. Comput Mater Sci 2009; 47: 712-20.
[14] Song PX, Wen DS. Molecular dynamics simulation of a core-shell structured metallic nanoparticle. J Phys Chem C 2010; 114: 868896.

[15] Kuntova Z, Rossi G, Ferrando R. Melting of core-shell Ag-Ni and Ag-Co nanoclusters studied via molecular dynamics simulations. Phys Rev B Condens Matter 2008; 77 :

[16] Henz BJ, Hawa T, Zachariah M. Molecular dynamics simulation of the kinetic sintering of $\mathrm{Ni}$ and $\mathrm{Al}$ nanoparticles. Mol Simul 2009; 35: 804-11.

[17] Evteev AV, Levchenko EV, Belova IV, Murch GE. Interdiffusion and surface-sandwich ordering in initial Ni-core-Pd-shell nanoparticle. Phys Chem Chem Phys 2009; 11: 3233-40.

[18] Nguyen NH, Hu A, Persic J, Wen JZ. Molecular dynamics simulation of energetic Aluminum/Palladium core-shell nanoparticles. 2010; (accepted).

[19] Gale JD, Rohl AL. The general utility lattice program (GULP). Mol Simul 2003; 29: 291-341.

[20] Cleri F, Rosato V. Tight-binding potentials for transition-metals and alloys. Phys Rev B Condens Matter 1993; 48: 22-33.

[21] Margolis SB, Green RM. Theoretical prediction of self-propagating, diffusion-limited and decomposition-limited intermetallic reactions. Combust Flame 1979; 34: 245-52.

[22] $\mathrm{Hu} \mathrm{RX}, \mathrm{Su} \mathrm{HN}$, Nash P. Enthalpies of formation and lattice parameters of B2 phases in Al-Ni-X systems. Pure Appl Chem 2007; 79: 1653-73.

[23] Ben-Naim A, Mountain R. Pair correlation functions in mixtures of Lennard-Jones particles. J Chem Phys 2008; 128: 214504.

[24] Ogata S, Iyetomi H, Tsuruta K, et al. Role of atomic charge transfer on sintering of $\mathrm{TiO} 2$ nanoparticles: Variable-charge molecular dynamics. J Appl Phys 2000; 88: 6011-5.

[25] Song PX, Wen DS. Molecular dynamics simulation of the sintering of metallic nanoparticles. J Nanopart Res 2010; 12: 823-9. 